\title{
Cell-specific histone modifications in atherosclerosis (Review)
}

\author{
WANLIN JIANG, DEVENDRA K. AGRAWAL and CHANDRA S. BOOSANI
}

Department of Clinical and Translational Science, Creighton University School of Medicine, Omaha, NE 68178, USA

Received January 31, 2018; Accepted May 21, 2018

DOI: $10.3892 / \mathrm{mmr} .2018 .9142$

\begin{abstract}
Histone modifications are the key epigenetic mechanisms that have been identified to regulate gene expression in many human diseases. However, in the early developmental stages, such as in utero and the postnatal stages, histone modifications are essential for gene regulation and cell growth. Atherosclerosis represents a classical example of the involvement of different cell types, and their cumulative effects in the development of atheroma and the progression of the disease. Post translational modifications on proteins either induces their functional activity or renders them inactive. Post translational modifications such as methylation or acetylation on histones have been well characterized, and their role in enhancing or inhibiting specific gene expression was clearly elucidated. In the present review article, the critical roles of different histone modifications that occur in atherosclerosis have been summarized. Different histone proteins have been identified to serve a critical role in the development of atherosclerosis. Specifically, histone methylation and histone acetylation in monocytes, macrophages, vascular smooth muscle cells and in endothelial cells during the progression of atherosclerosis, have been well reported. In recent years, different target molecules and genes that regulate histone modifications have been examined for their effects in the treatment of atherosclerosis in animal models and in clinical trials. An increasing body of evidence suggests that these epigenetic changes resulting from DNA methylation and non-coding RNA may also be associated with histone modifications, thereby indicating that novel therapeutic strategies can be developed by targeting these post translational modifications, which may in turn aid in the treatment of atherosclerosis.
\end{abstract}

Correspondence to: Dr Chandra S. Boosani, Department of Clinical and Translational Science, Creighton University School of Medicine, 2500 California Plaza, Omaha, NE 68178, USA

E-mail: chandraboosani@creighton.edu

Key words: atherosclerosis, foam cell, histone acetylation, histone methylation, macrophages, monocytes, endothelial cells, smooth muscle cells, plaque, epigenetic, non-coding RNA

\section{Contents}

1. Introduction

2. Histone methylation

3. Histone acetylation

4. Targeting histone methylation and histone acetylation in the treatment of atherosclerosis

5. Future directions in targeting histone methylation and histone acetylation in the treatment of atherosclerosis

\section{Introduction}

During the progression of atherosclerosis, oxidized low-density lipoprotein (oxLDL) initiates endothelial dysfunction and increases the expression of vascular cell adhesion molecule-1 (VCAM-1), P-selectin, and chemokine monocyte chemoattractant protein-1 (MCP-1) that recruits monocytes, T-lymphocytes and platelets. Monocytes are stimulated by monocyte-colony stimulating factor (M-CSF), and pro-inflammatory chemokines and cytokines, which differentiate them into macrophages. Macrophages then engulf oxLDL by receptor-mediated phagocytosis and transform themselves into lipid-laden foam cells. Accumulated foam cells and immune cells in the vessel wall develop an identifiable pathological condition called, the 'fatty streak'. This first symptom represents asymptomatic and non-stenotic plaque, and is the early sign for the development of atherosclerosis. Subsequently, the smooth muscle cells (SMCs) triggered by cytokines, chemokines, and growth factors, gain the ability to proliferate and migrate to develop a fibrous cap covering the atherosclerotic core. In this advanced pathological stage of atherosclerosis, matrix degradation, cytotoxic T-cells and cholesterol crystals accumulate to form a lipid-rich necrotic core. In the very late stage of atherosclerosis, apoptosis in the SMCs of the fibrous cap occurs which causes plaque instability and thrombosis. As a result, distal embolism occurs which leads to blockage in the cerebral arteries. Thus, atherosclerosis in coronary arteries and in carotid arteries results in coronary heart disease and ischemia in the brain which in severe conditions may cause significant morbidity and mortality (1). During the progression of atherosclerosis from 'fatty streak' to atheroma development, changes in the gene expression in different cell types have been reported. Below, we describe the de novo epigenetic changes, specifically histone modifications which were reported in 
different cell types that are involved in the development of atherosclerosis.

\section{Histone methylation}

DNA normally remains coiled to the histones proteins ( $\mathrm{H} 1$, H2A, H2B, H3 and H4) in eukaryotes systems. Tight control over gene expression occurs accurately and effectively via chromatin compaction or signaling, or by activating other signaling events such as phosphorylation, acetylation, ubiquitination and methylation on histones. These modifications on the histone proteins are collectively referred to as histone post-translational modifications (PTMs). Since these histone modifications have been identified to either induce or inhibit transcription, their role in regulating gene expression, which is specific to each type of modification on different histones, has been well studied $(2,3)$.

Histone proteins are predominantly methylated on arginine and lysine residues. Multiple modifications can occur on these histone proteins such as mono-methylation (me1), di-methylation (me2) or tri-methylation (me3). The Su(var)3-9, Enhancer-of-zeste and Trithorax domain containing proteins (SET-domain-containing proteins), Disruptor of telomeric silencing 1-like (DOT1-like proteins), and Calmodulin-lysine $N$-methyltransferase are three families of proteins which transfer methyl groups from $S$-adenosyl methionine to histones. On the contrary, the two family of proteins which function as demethylases have also been identified which include amine oxidases and jumonji C (JmjC)-domain-containing proteins, and iron-dependent dioxygenases (4).

Histone methylation as a prognostic marker in atherosclerosis. Methylation on $\mathrm{H} 3 \mathrm{~K} 4$ has been correlated with stage-specific progression of atherosclerosis mediated by GCN5L and MYST1, MLL2/4 proteins (3). In ApoE-/-mice, high levels of homocysteine (Hcy) induced EZH2 expression was detected which led to $\mathrm{H} 3$ at lysine 27 (H3K27) tri-methylation (5). The polycomb complex protein, BMI-1, is one of the proteins in PRC1-like complex, which is required to inhibit the expression of target genes through histone modification such as mono-ubiquitination on H2AK119. As part of PRC1 complex, BMI-1 promotes E3 ubiquitin-protein ligase activity (6). Global increase in trimethylation of $\mathrm{H} 3 \mathrm{~K} 27$ was observed in atherosclerotic plaques at late stage in the pathology. However, decreased trimethylation of H3K27 was not associated with either BMI-1, histone methyltransferase EZH2, or histone demethylase JMJD3, which targets H3K27 (7). Increased expression of H2AK119Ub and H2BK120Ub has been reported in diabetic cardiomyopathy, and treatment with Esculetin was identified to mitigate the renin-angiotensin system, oxidative stress (Keap1) and cell proliferation (Ki67) via $\mathrm{H} 2 \mathrm{~A} / \mathrm{H} 2 \mathrm{~B}$ ubiquitination, which eventually attenuated metabolic alterations, hypertension, cardiomyocytes hypertrophy, and fibrosis in the hearts of type 2 diabetic rats (8). The S-Adenosyl homocysteine (SAH) level in the plasma holds a strong correlation with the size of atherosclerotic lesion and in the pathogenicity of hyperhomocysteinemia during atherosclerosis. The increased levels of SAH in plasma was reported to promote expression of glucose-regulated protein-78 and CEBP-homologous protein, which are indicators of endoplasmic reticulum stress resulting from the repression of trimethylation on histone $\mathrm{H} 3$ at lysine 9 (H3K9) (9). The histone-lysine N-methyltransferase (MLL5) activity, which serves an important role in cell cycle regulation, and SNP variations at this locus, have been attributed with coronary artery disease. The dominant genotype of rs12671368 and the recessive genotype of $\mathrm{rs} 2192932$ were found to provide protective effects in a study that was carried out on Chinese Han population (10).

Monocytes and macrophages. One of the primary functions of G9a is to mono-methylate and di-methylate H3K9 by forming G9a-GLP (G9a-like protein) complex, which plays a critical role in the regulation of gene expression in monocytes (11). In hyperhomocysteinemic ApoE-/-mice that were treated with methionine, decreased expression of G9a as well as $\mathrm{H} 3 \mathrm{~K} 9$ di-methylation was reported to promote apoptosis in the macrophages affecting the foam cell formation and also plaque stability (12). Inhibiting trimethylation of $\mathrm{H} 3 \mathrm{~K} 4 \mathrm{me} 3$ and $\mathrm{H} 3 \mathrm{~K} 27 \mathrm{me} 3$ was found tightly associated with increased expression of specific marker genes such as KLF4, IRF8, HOXA and FOXO which stimulate monocyte-into-phagocyte (MP) differentiation (13). In monocytes, reduced methylation on H3K9 and H3K27 was observed in inflammatory cells (14). Monocytes typically gets reprogramed into pro-inflammatory phenotype by oxLDL, and this was found to increase during aerobic glycolysis. Analysis of the monocytes from patients with asymptomatic and symptomatic carotid plaques revealed that monocytes from patients with symptomatic carotid plaque expressed more pro-inflammatory cytokines after stimulating with LPS. Lower levels of H3K4me3 and H3K27me3 were found on the promotor region of TNF- $\alpha$. At the same time, reduced glycolytic rate limiting enzymes hexokinase 2 and 6-phosphofructo-2-kinase/fructose-2,6-biphosphatase 3 expressions were detected in the carotid plaques of symptomatic compared to asymptomatic patients with carotid stenosis (15). The suppressor of variegation 3-9 homologue1 (SUV39H1) is a histone methyltransferase which mediates trimethylation at $\mathrm{H} 3 \mathrm{~K} 9$, and acetylation at lysine residue 266 was found to decrease its activity. SIRT1 was reported to recruit and deacetylate $\mathrm{SUV} 39 \mathrm{H} 1$, and this deacetylation of SUV39H1 contributes to the increased functional activity of SUV39H1 thereby, the levels of H3K9me3 will get elevated (16). In macrophages, high-glucose stimulation reduced trimethylation on $\mathrm{H} 3 \mathrm{~K} 9$ which was mediated through SUV39H1. This further led to increased expression of the inflammatory cytokines such as IL-6, IL-12, p40, MIP-1 $\alpha$, and MIP-1 $\beta$ (17). In peripheral blood monocytes (PBM) of patients with type 2 diabetes mellitus (T2DM), Set7 induced mono-methylation at $\mathrm{H} 3 \mathrm{~K} 4$ was found increased at the promotor of NF- $\mathrm{B}$ p65, leading to the activation of $\mathrm{NF}-\kappa \mathrm{B}$-dependent oxidative and inflammatory signaling. This in turn elevated the expression levels of the downstream pro-inflammatory genes ICAM-1 and MCP-1. Set7 was also found associated with the oxidative marker 8 -isoPGF $2 \alpha$ and brachial artery flow-mediated dilation (FMD) (18). The oxLDL, TLR-2 and TLR-4 agonists can induce human monocyte differentiation into foam cells by stimulating trimethylation of $\mathrm{H} 3 \mathrm{~K} 4$ at the promotor regions of different 
Table I. Histone methylation markers in monocytes and macrophages in atherosclerosis.

\begin{tabular}{llll}
\hline Epigenetic mediator & $\begin{array}{c}\text { Histone } \\
\text { modification }\end{array}$ & $\begin{array}{c}\text { Expression in } \\
\text { atherosclerosis }\end{array}$ & \multicolumn{1}{c}{ Target genes } \\
\hline G9a, SUV39H1 & H3K9 & Decreased & IL-6, IL-12p40, MIP-1 $\alpha$, and MIP-1 $\beta$ \\
Set7 & H3K4me1 & Increased & NF- $\kappa$ B p65 \\
& H3K4me3 & Decreased & HOXA, FOXO, KLF4, IRF8, TNF $\alpha$, IL-6, IL-18, \\
& H3K27me3 & Decreased & HOXA, FOXO, KLF4, IRF8 and TNF- $\alpha$ \\
\hline
\end{tabular}

SUV39H1, suppressor of variegation 3-9 homologue 1; H3K9, histone H3 at lysine 9; H3K4, histone H3 at lysine 4; H3K27, histone H3 at lysine 27; me 1/3, mono/tri-methylation; Set7, SET domain containing lysine methyltransferase 7; IL, interleukin; MIP, macrophage inflam-

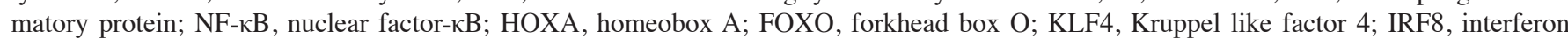
regulatory factor 8 ; TNF $\alpha$, tumor necrosis factor- $\alpha$; MCP-1, monocyte chemoattractant protein 1; MMP, matrix metalloproteinase; $\mathrm{CD}$, cluster of differentiation; SR-A, macrophage scavenger receptor 1.

genes such as IL-6, IL-18, TNF- $\alpha$, MCP-1, MMP2, MMP9, CD36, and SR-A (19). In Table I, we summarize histone modifications occurring on different genes in monocytes and macrophages that are regulated during atherosclerosis.

Endothelial cell. TNF- $\alpha$ stimulates the expression of ICAM1 which causes leukocyte adhesion to endothelial cells via reducing methylation of $\mathrm{H} 3 \mathrm{~K} 9$ and $\mathrm{H} 3 \mathrm{~K} 27$ residues. Overexpression of G9a or inhibition of KDM4D can increase the expression of $\mathrm{H} 3 \mathrm{~K} 9 \mathrm{me} 2$ and reduce adhesion of leukocytes to endothelial cells (20). In endothelial cells, SAH which is a potent inhibitor of S-adenosylmethionine-dependent methyltransferases, was found to induce endothelial cell activation by increasing the expression of adhesion molecules and cytokines, by stimulating $\mathrm{NF}-\kappa \mathrm{B}$ pathways. SAH reduced the expression of EZH2 and tri-methylation of H3K27 (21). In endothelial cells, TNF- $\alpha$ increased the expression of histone demethylases KDM1B and KDM7A which further induced H3K9me2 di-methylation. KDM7A mediated upregulation of ICAM1 was found associated with decreased expression of the transcription factor EB which positively regulates the activity of lysosome formation (22). Arginine methyltransferase inhibitors, AMI-5 and AMI-1, inhibit migration of endothelial cells to prevent pathological angiogenesis by stimulating the formation of heterochromatin. This was reported to be mediated through downregulation of arginine and lysine histone methyltransferases (HMTs) (23). PHD finger protein 8 (PHF8) was shown to induce endothelial cell migration and formation of capillary-like structures by decreasing di-methylation of E2F4, suggesting its functions as a repressor (24). In human umbilical vein cell line EA.hy926, elevated di- and tri-methylation (H3K4me2/3) on MCP-1 promotor was found regulated by increased $\mathrm{H} 3 \mathrm{~K} 4$ histone methyltransferase MLL3, menin and SET7 along with a concomitant decrease in the demethylase LSD1 expression in high glucose conditions. Puerarin was also reported to decrease the expression of MCP-1 by reversing the expression of methyltransferases and demethylases (25). During the progression of atherosclerosis, a subset of dendritic cells also takes up the lipid molecules and this leads to the formation of foam cells. Elevated cholesterol levels in plasma indicates the onset of atherosclerotic phenotype in T-cells compared to anti-atherosclerotic phenotype in normal group (26). In human aortic endothelial cells (HAECs), Set7 was found to stimulate $\mathrm{NF}-\kappa \mathrm{B}$-dependent oxidative and inflammatory signaling by mono-methylation of $\mathrm{H} 3 \mathrm{~K} 4$ at its promotor (18). In Endothelial cells, EZH2 was reported to be induced by LDL. This was observed to be mediated through the transcription activator, myocyte enhancing factor-2, which decreased the expression of KLF2. The decreased expression of KLF2 was found to affect the expression of different cellular regulators of atherosclerosis such as thrombomodulin, endothelial NO synthase, and plasminogen activator inhibitor-1, which promote accumulation of platelets on endothelial cells (27). 'Hyperglycemic memory' is a condition which induces deleterious effects in patients even after more than five years of the onset of glycemic control. In bovine aortic endothelial cells, hyperglycemia induced expression of NF-кB-p65 was found to upregulate the expression of $\mathrm{H} 3 \mathrm{~K} 4 \mathrm{~m} 1$, and this was associated with the reduced expression of di- and tri-methylation of histone $\mathrm{H} 3$ (H3K9m 2 and $\mathrm{H} 3 \mathrm{~K} 9 \mathrm{~m} 3)$. This reduction in histone methylation was identified as mediated through histone demethylase LSD1 $(28,29)$. Hyperglycemia also stimulated the expression of p65 in aortic endothelial cells, which induced the expression of MCP-1 and VCAM-1. This suggests that by decreasing the levels of mitochondrial superoxide production or superoxide-induced $\alpha$-oxoaldehydes, inflammation under hyperglycemic condition can be prevented (29). In Table II, we summarized the histone modifications occurring exclusively in the endothelial cells during atherosclerosis, affecting different target gene loci.

Vascular SMCs. Under in vitro conditions, high glucose increases the expression of KDM3a, causing reduction in H3K9 di-methylation at AGTR1 and ROCK2 loci. This indicates that the proliferation and migration of vascular SMCs (VSMCs) is enhanced through activation of Rho/ROCK and AngII/AGTR1 pathways (30). Increased histone acetylation at $\mathrm{H} 3 \mathrm{~K} 9$ and $\mathrm{H} 3 \mathrm{~K} 27$ led to a parallel decrease in the methylation at $\mathrm{H} 3 \mathrm{~K} 9$ and $\mathrm{H} 3 \mathrm{~K} 27$ which was found in the advanced atherosclerotic lesions (14). VSMCs upon treatment with Acrolein, which is an air pollutant and consists of cigarette smoke, was identified to induce histone acetylation at H3K9 and H3K56. Simultaneous decrease in the histone tri-methylation at $\mathrm{H} 3 \mathrm{~K} 9$ 
Table II. Histone methylation markers in endothelial cells in atherosclerosis.

\begin{tabular}{llll}
\hline Epigenetic mediator & Histone modification & Expression in atherosclerosis & Target genes \\
\hline G9a & H3K9m2 & Decreased & ICAM1 \\
KDM4D & H3K9m2 & Increased & ICAM1 \\
KDM7A & InK9m2 & Increased & ICAM1 \\
PHF8 & Demethylation & Increased & E2F4 \\
MLL3 & H3K4m2, H3K4m3 & Decreased & MCP-1, p65 \\
LDS1 & H3K4m2, H3K4m3 & & MCP-1, p65 \\
& H3K9m2, H3K9m3 & Increased & NF-kB \\
SET7 & H3K4m1 & Increased & KLF2 \\
\hline
\end{tabular}

$\mathrm{H} 3 \mathrm{~K}$ 9, histone $\mathrm{H} 3$ at lysine 9; H3K4, histone $\mathrm{H} 3$ at lysine 4; MCP-1, monocyte chemoattractant protein 1; NF- $\mathrm{B}$, nuclear factor- $\kappa \mathrm{B}$; KLF2, Kruppel like factor 2; KDM 4D/7A, lysine demethylase 4D/7A; PHF8, PHD finger protein 8; MLL3, mixed-lineage leukemia protein 3; LDS1, Loeys-Dietz syndrome 1; SET7, SET domain containing lysine methyltransferase 7; EZH2, enhancer of zeste homolog 2; ICAM1, intercellular adhesion molecule 1; E2F4, E2F transcription factor 4.

was also observed. Co-culturing with $\mathrm{N}$-acetyl cysteine has been shown to inhibit the toxicity of acrolein. In VSMCs this was correlated with significant decrease in histone acetylation at $\mathrm{H} 3 \mathrm{~K} 9$. At the same time, other histone modifications were also observed such as di-methylation at $\mathrm{H} 3 \mathrm{~K} 4$, and phosphorylation and di-methylation at H3S10 (31). In VSMCs, treatment with Roflumilast, which is a PDE4 inhibitor, was reported to affect TNF- $\alpha$ functions through VCAM-1 by promoting the expression of the cyclic AMP effector, Epac. Upregulation of Epac subsequently reduced histone di-methylation H3K4me2 at VCAM-1 and promoted its expression. This epigenetic action of Roflumilast on VCAM-1 can be reverted by treatment with an HDAC inhibitor (32). High-glucose conditions were also reported to increase JMJD2A expression in the VSMCs of the rat carotid arteries which enhanced cell proliferation. Tri-methylation of H3K9 was inversely correlated with JMJD2A expression in VSMCs of the balloon-injured arteries of diabetic rats where histone methylation suppressed the expression of MCP-1 and IL-6. Thus, inhibition of JMJD2A appears to be a potential treatment option to prevent inflammation in VSMCs (33). Moreover, global reduction in the levels of $\mathrm{H} 3 \mathrm{~K} 27$ in advanced atherosclerotic plaques did not hold any correlation with the expression of histone methyltransferase, $\mathrm{EZH} 2$, which is the catalytic component of the polycomb repressive complex 2 (PRC2). Further, BMI1 which consists of PRC1 complex, promoted tri-methylation of $\mathrm{H} 3 \mathrm{~K} 27$ and JMJD3 which is a histone demethylase that targets H3K27. The reduction in $\mathrm{H} 3 \mathrm{~K} 27 \mathrm{Me} 3$ tri-methylation in the tunica media also plays an important role in the differentiation and proliferation of VSMCs in atherosclerosis (7). Decreased expression of Suv39h1 would lead to reduced levels of H3K9me3 in VSMCs in type 2 diabetic $\mathrm{db} / \mathrm{db}$ mice when compared with control $\mathrm{b} /+$ cells, suggesting that VSMCs are hypersensitive to TNF- $\alpha$ stimulation. Moreover, the corepressor HP1 $\alpha$ was also reported to be decreased in $\mathrm{db} / \mathrm{db}$ cells (34).

Upregulation of major histocompatibility complex II (MHC II) molecules in VSMCs, which recruit T lymphocyte and stimulate the expression of inflammatory cytokines, has been well characterized in the pathogenesis of atherosclerosis.
By stimulating A2b adenosine signaling pathway, PCAF/GCN5 which are histone H3K9 acetyltransferases, and WDR5 which consists of $\mathrm{H} 3 \mathrm{~K} 4$ methyltransferase complex, prevents their recruitment at the promotor region of CIITA transcription, thereby inhibiting the expression of CIITA in a STAT1-dependent manner (35). Specific methylation patterns in histone proteins that have been reported in the VSMCs during atherosclerosis was summarized in Table III.

In utero programming and postnatal histone methylation in atherosclerosis. Maternal hypercholesterolemia could have direct impact in inducing atherosclerosis in the offspring as reported in ApoE-/-Leiden mice (36). Compared with the offspring from wild-type mothers, the offspring from ApoE-/-mothers showed differences in histone tri-methylation in endothelial cells and VSMCs, which caused different responses to high cholesterol diet. This study highlights the importance of in-utero programming and postnatal histone modification in atherosclerosis which if addressed in early stages may help in treating the disease (37). In Fig. 1, we summarize the histone methylation in different cell types and their effects on regulating gene expression during atherosclerosis.

\section{Histone acetylation}

Histone acetylation is regulated by two key enzymes, histone acetyltransferases (HATs) and histone deacetylases (HDAC). By transferring an acetyl group to lysine side chains of histone proteins, HATs weaken the binding between histone and DNA. On the contrary, HDACs remove the acetyl group on the histones to increase the interaction between histone and DNA. HATs are further categorized into two groups, type-A and type-B (38). HDACs regulate transcription and cell cycle progression by deacetylating lysine residues located on the $\mathrm{N}$-terminal of the core histone proteins $(\mathrm{H} 2 \mathrm{~A}, \mathrm{H} 2 \mathrm{~B}, \mathrm{H} 3$ and H4). HDACs are further classified into four Classes I, II, III and IV. Classes I, II and IV represent 'classical' HDACs which include 11 genes, and in Class III, 7 genes are included 
Table III. Histone methylation markers in vascular smooth muscle cells in atherosclerosis.

\begin{tabular}{lccc}
\hline Epigenetic mediator & Histone modification & Expression in atherosclerosis & Target genes \\
\hline KDM3a & H3K9 & Increased & AGTR1, ROCR2 \\
Epac & H3K4m3 & ICAM-1 \\
JMJD2A & H3K9 & Increased & MCP-1, IL-6 \\
EZH2 & H3K27 & Increased & - \\
SUV39H1 & H3K9m3 & Decreased & TNF- $\alpha$ \\
\hline
\end{tabular}

H3K9, histone $\mathrm{H} 3$ at lysine 9; $\mathrm{H} 3 \mathrm{~K} 4$, histone $\mathrm{H} 3$ at lysine 4; H3K27, histone $\mathrm{H} 3$ at lysine 27; ICAM1, intercellular adhesion molecule 1; IL-, interleukin; MCP-1, monocyte chemoattractant protein 1; TNF- $\alpha$, tumor necrosis factor- $\alpha$; AGTR1, angiotensin II receptor type 1; ROCR2, arginine utilization regulatory protein RocR; KDM3a, lysine demethylase 3A; Epac, exchange factor directly activated by cyclic adenosine monophosphate; JMJD2A, jumonji domain-containing protein 2A; EZH2, enhancer of zeste 2 polycomb repressive complex 2 subunit; SUV39H1, suppressor of variegation 3-9 homolog 1.

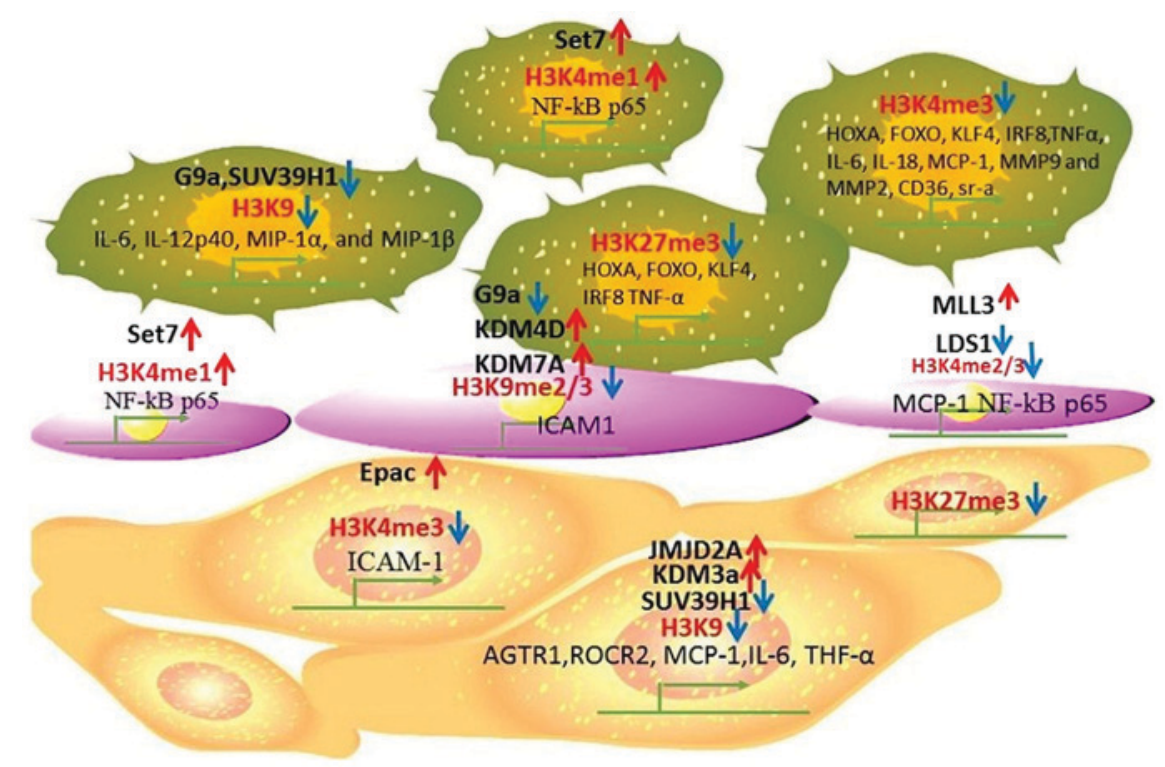

Figure 1. Histone methylation occurring during atherosclerosis. Methylation of different histone proteins and their effects on gene expression in various cell types during atherosclerosis is presented. Red arrows indicate upregulation and blue arrows indicate downregulation.

which are named as sirtuins (39). Based on the type of modification occurring on the histones, gene expression is either favored or inhibited. This characteristic feature of the PTMs on histones and their role in transcriptional regulation is developmental and tissue specific. However, such histone modifications, to regulate gene expression, are frequently observed in many pathological conditions. Such modifications occurring in atherosclerosis are discussed below.

Histone acetylation as an indicative marker in atherosclerosis. In the heart tissues of New Zealand white rabbits that were treated with high cholesterol diet and atorvastatin, the mRNA levels of ACE2 was reported to be elevated when compared with control high cholesterol diet. Notably, atorvastatin was identified to promote histone $\mathrm{H} 3$ acetylation (H3-Ac) at the promoter region of ACE2 (40). High plasma levels of Hcy was identified not only to increase abnormal DNA methylation in the cells but also elevates the levels of N-methyl-d-aspartate receptor-1 (NMDAR1), DNA (cytosine-5)-methyltransferase-1 (DNMT1) and MMP-9, via acetylation on H3K9 and by inhibiting the expression of HDAC1. These regulatory proteins were identified as possible targets that have high potential to cause heart failure as reported in cardiomyocytes (41).

Monocytes and macrophages. The 'Silent mating type information regulator two homologue one' (SIRT1), which belongs to class III HDAC, requires Nicotinamide adenine dinucleotide (NAD+) as a cofactor for its functional activity. The role of SIRT1 has been implicated in many cellular processes such as in cell cycle progression, inflammation, DNA damage, apoptosis, autophagy, aging as well as in metabolic disease (42). SIRT1 mediates transfer of the acetyl group from proteins to the targeting co-substrate by removing the nicotinamide ribosyl bond of NAD+. In the peripheral blood mononuclear cells (PBMCs) of patients with T2DM, increased transcription of HDAC3 was reported. SIRT1, which was identified as a protective marker of cardiovascular diseases, was found reduced in PBMCS of these T2DM patients. In addition, the pro-inflammatory markers TNF- $\alpha$, IL-6, MCP-1, IL1- $\beta$, NF $\kappa$ B, TLR2, and TLR4 were also reported to be upregulated in the 
Table IV. Histone acetylation markers in monocytes and macrophages in atherosclerosis.

\begin{tabular}{lcl}
\hline Enzyme & $\begin{array}{c}\text { Expression in } \\
\text { atherosclerosis }\end{array}$ & \multicolumn{1}{c}{ Target genes } \\
\hline HDAC7 & Increased & IL-6, MCP-1 \\
P300 & Decreased & Transcription regulation \\
HDAC9 & Increased & MMP12; ABCA1, \\
& & ABCG1, PPAR- $\gamma$ \\
\hline
\end{tabular}

HDAC, histone deacetylase; IL, interleukin; MCP-1, monocyte chemoattractant protein 1; ABCA1, adenosine triphosphate-binding cassette subfamily A member 1; adenosine triphosphate-binding cassette subfamily $G$ member 1; PPAR- $\gamma$, peroxisome proliferator-activated receptor- $\gamma$.

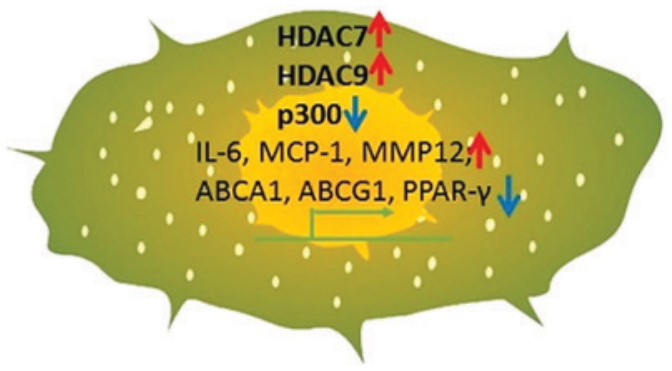

Figure 2. Histone acetylation occurring during atherosclerosis. Acetylation of different histone proteins and their effects on regulating gene expression in monocytes and macrophages during atherosclerosis is presented Red arrows indicate upregulation and blue arrows indicate downregulation. HDAC, histone deacetylases; IL-6, interleukin 6; MCP-1, chemokine monocyte chemoattractant protein-1; MMP12, matrix metalloproteinase 12; ABCA1, adenosine triphosphate binding cassette subfamily A member 1; ABCG1, adenosine triphosphate binding cassette subfamily $\mathrm{G}$ member 1 PPAR- $\gamma$, peroxisome proliferator-activated receptor- $\gamma$.

plasma of the T2DM patients. Further, DBC1 (deleted in breast cancer 1), which represses HDAC3, was also reported to be decreased in the PBMCs of patients with T2DM (43). Myeloid HDAC3 deficiency was reported to have plaque stabilization effect by increasing the deposition of collagen, HDAC3 expression can potentially induce macrophage polarization, favoring the formation M2 phenotypes. These M2 macrophages by secreting TGF $\beta 1$ were shown to initiate VSMCs to secrete excess collagen $(44,45)$ Under hypercholesterolemic conditions, the orphan nuclear receptor NR4A1, which can effectively reduce IL- 6 and MCP-1 expression, was found increased due to the recruitment of p300 acetyltransferase. As a result, HDAC7 (histone deacetylase 7) was found repressed which contributes to the expression of NR4A1 in human monocytes, THP-1 and U937 (46). The histone deacetylase 9, HDAC9, was reported to be associated with MMP12 expression in the pro-inflammatory macrophages M2 and M4, in the advanced human plaques. However, the presence of both these types of macrophages could not be correlated with genes regulating plaque stabilization or thrombosis (47). HDAC9 deficient cells were identified to express increased levels of ABCA1, ABCG1, and PPAR- $\gamma$. This was identified to be mediated through upregulation of $\mathrm{H} 3$ and $\mathrm{H} 4$ acetylation, preventing the cholesterol efflux. Moreover, HDAC9 knockout macrophages were identified to be secreting lower levels of pro-inflammatory cytokines and showed prominent expression of M2 phenotype markers, and at the same time, it simultaneously decreased the expression of M1 markers $(48,49)$. In Table IV, we summarized acetylation events on different residues of histones in the above monocytes and macrophages occurring during atherosclerosis. The same was represented in Fig. 2.

Othercell types. The protein 'General Control Non-repressible 5' (GCN5)-related N-acetyltransferase (GNAT), mediates transfer of the acyl group from acyl coenzyme A (acyl-CoA) to different proteins substrates which are involved in transcription, cell proliferation, antioxidant functions, antibiotic resistance and detoxification (50). The p300/CREB-binding protein (CBP) which is a HAT, acetylates non-histone proteins and controls transcription and DNA repair mechanisms in the cells (51). When compared between the cardiac mesenchymal stem cells from normoglycemic subjects, cardiac mesenchymal stem cells from type 2 diabetic patients had decreased levels of H3K9Ac and H3K14Ac acetylations, which are caused primarily due to the reduced activity of GCN5-related N-acetyltransferases (GNAT) p300/CBP-associated factor. Also its isoform, GCN5a, decreased proliferation and differentiation of MSCs. Moreover treatment with the HAT activator, pentadecylidenemalonate 1b (SPV106) not only recovered the levels of H3K9Ac and H3K14Ac acetylation, but also decreased CpG hypermethylation in the genomic DNA, suggesting the involvement of two different epigenetic mechanisms regulating at the same time in these cells (52).

\section{Targeting histone methylation and histone acetylation in the treatment of atherosclerosis}

As cell specific histone modifications have been identified during atherosclerosis, targeting the enzymes which regulate these de novo changes can be a promising strategy for the treatment of atherosclerosis. This strategy is favored by two important features. Frist, like all epigenetic therapies, targeting histone modification alone do not affect the genetic component in the cells. Second, advances in the structural biology greatly assists the researchers to develop unique compounds that can specifically target the desired histone modification, such as the DNMT inhibitor (2'-deoxy-5-azacytidine, DAC) which targets $\mathrm{H} 3 \mathrm{~K} 9$ dimethylation, and the HDAC inhibitor TSA, which targets H3K27 trimethylation (53). Since any given histone modification is not unique for a specific disease and as they can be observed in different pathologies, drugs targeting a specific histone modification can be repurposed for other disease conditions. In addition, use of these specific histone targeting drugs as combination therapy with other therapeutic drugs appears to be more promising.

Treatment strategies targeting histone methylation. The selective inhibitor, 3-deazaneplanocin A (DZNep) prevents methylation at lysine 27 on histone H3 (H3K27me3) and lysine 20 on histone $\mathrm{H} 4$ (H4K20me3) is currently used in the treatment of cancer (54). Upregulation of Lysine methyltransferases (KMTs) plays an important role in the differentiation of monocytes into immature dendritic cells (iDCs). Two of the 
know drugs that inhibit histone methylation, BIX-01294 which is KMT1c inhibitor, and DZNep, prevents global KMT activity were found to inhibit monocytes differentiating into iDCs (55).

Treatment strategies targeting histone acetylation. HDAC1 expression was identified to decrease acetylation of $\mathrm{H} 3 \mathrm{~K} 9 \mathrm{ac}$ which induces accumulation of total cholesterol, free cholesterol, and triglycerides in foam cells, as seen in the aorta of ApoE-/-mice, which was abrogated by upregulation of the microRNA miR-34a (56). The compound 4-hydroperoxy-2-decenoic acid ethyl ester (4-HPO-DAEE) isolated from the royal jelly was found to acetylate histone $\mathrm{H} 3$ and $\mathrm{H} 4$ at the proximal promoter region of EC-SOD (extracellular superoxide dismutase) in THP-1 cells, which is considered as a potential target for the treatment of atherosclerosis (57). Treatment with histone deacetylase inhibitor, valproic acid, was shown to promote the release of endogenous t-PA in males with vascular disease (58). Several HDAC inhibitors are used in the current standard of care. The known synthetic HDAC inhibitors which inhibit Class III HDACs include; dihydrocoumarin, naphthopyranone, 2-hydroxynaphaldehydes and other sirtuin-inhibiting agents. Class I, II, and IV HDAC inhibitors were grouped as hydroxamates such as trichostatin A (TSA), vorinostat (suberoylanilide hydroxamic acid; SAHA), belinostat (PXD101), panobinostat (LBH589), LAQ824, peptide inhibitors such as cyclic tetrapeptides (trapoxin B) and depsipeptides, benzamides (entinostat (MS-275), mocetinostat (MGCD0103), CI994, aliphatic acid components (phenylbutyrate, valproic acid), and electrophilic ketones (51). Some of the plant polyphenols such as curcumin (diferuloylmethane) and resveratrol were identified as naturally available HDAC inhibitors.

\section{Future directions in targeting histone methylation and histone acetylation in the treatment of atherosclerosis}

Several histone modifications regulating the off-target drug effects have been reported which indicates their additional benefits. Both Hydralazine and Osalazine which are used to treat hypertension can be repurposed for treating neoplasia as their treatment can restore the functions of tumor suppressor genes. As a histone-deacetylase inhibitor, Romidepsin when used in endometriosis, efficiently induced apoptosis in the epithelial cells of endometrium. However, off-targets effects on histone modification can potentially exert negative effects in the cells. Similarly, the histone deacetylase inhibitor, valproic acid which is prescribed for epilepsy treatment, was identified as a causative factor for spina bifida in the growing embryos (59). Thus, for future considerations, specific mechanisms of histone modifications should be determined in patients for prognosis to avoid adverse drug effects.

DNA methylation intercepts histone modifications. During the early developmental stages of the embryo, unmethylated CpG islands were found to induce the expression of DNMT3A and DNMT3B. At the same time, DNMT3L interacts with the chromatin and targets $\mathrm{H} 3 \mathrm{~K} 4$. G9a was found to methylate H3K 9 and recruit heterochromatin protein 1, DNMT3A and DNMT3B during stem cell production (60). Existence of such epigenetic cross connections may also lead to de novo gene regulations which can be critically involved in the disease progression.

Non-coding RNA and histone modification. Another key epigenetic mechanism that was found to intercept histone modifications is the expression of long non-coding RNAs. Under hypoxic conditions, upregulated expression of HDAC3 was reported to inhibit the expression of long non-coding RNA 'IncRNA-LET' (61). During the phenotype switch in VSMCs, the long non-coding RNA 'taurine up-regulated gene-1' (TUG1), was found to form a hetero-complex with EZH2 to methylate $\alpha$-actin. As a result, the levels of $\alpha$-actin were found decreased, and simultaneously F-actin was elevated. This imbalance was presumed to be a causative factor for the phenotype switch in the VSMCs which change from contractile to synthetic phenotype (62). In lung cancer, the long non-coding RNA, MALAT1, was reported to upregulate the expression of histone demethylase JMJD1A, which was known to promote cell migration and invasion (63).

Hyperlipidemia and hypertension. Several factors such as hyperlipidemia, hypertension, diabetes and also diet play an important role in the development and progression of atherosclerosis. The onset of de novo molecular mechanisms which are discussed above indicates epigenetic mechanisms to be critically involved in the pathogenesis. It was reported that high-fat diet decreases ATP citrate-lyase levels as well as acetyl-CoA and/or the ratio of acetyl-CoA:CoA in white adipose tissue and pancreas, leading to inhibition of $\mathrm{H} 3 \mathrm{~K} 23$ acetylation in mice fed with high-fat diet (64). Dyslipidemia can cause monocyte priming such as increase in the adhesion of monocytes. Chemotaxis in the monocytes was promoted due to reduction in the H3K27 acetylation in non-human primates (65). H3K4 methylation of lysine-specific demethylase-1 (LSD1), H3K6 methylation of dehydrogenase 2 (HSD11B2), H3K79 methylation on epithelial sodium channel subunit $\alpha$ (SCNN1A) were also shown to be associated with arterial hypertension (66).

Clinical significance of HDAC inhibitors for the treatment of atherosclerosis. Panobinostat is a non-specific HDAC inhibitor that has been shown to have nanomolar potency, and can effectively inhibit Class I, II and IV HDACs. It induces apoptosis primarily by activating caspases and inducing PARP cleavage. In a phaseI/II clinical trial on 15 HIV positive patients, treatment with an HDAC inhibitor, panobinostat, was found to lower cardiovascular biomarkers such as CRP, LDL receptor, MCP1, E-Selectin, and HMGB1 in addition to lowering several inflammatory markers (67).

Trichostatin A, is a nonspecific inhibitor of Class I and Class II HDACs. Although it has been identified to prevent inflammatory markers such as IL-1b and IL-6, and have significant anti-tumor properties, it appears to have a more deleterious role in atherosclerosis. Treatment with Trichostatin A was found to promote proatherogenic markers such as TNF- $\alpha$, SRA, CD36, eNOS and VCAM-1 (68). However, Trichostatin A was found to inhibit p21 mediated proliferation of VSMCs (69). These reports suggest that Trichostatin A may have a role in preventing neointimal hyperplasia or maintaining plaque stability. Trichostatin A treatment upregulates the expression 
of Arginase 2 enzyme which promotes eNOS expression, inflammation and cell proliferation during progression of atherosclerosis. Earlier, it was shown that HDAC2 directly binds to the Arginase 2 promoter and inhibits its expression. In the same study, treatment with the HDAC2 specific inhibitor 'mocetinostat', was shown to enhance oxLDL induced endothelial dysfunction (70).

Though certain HDAC inhibitors have shown promising results in treating cancer and other diseases, the same had a different role in atherosclerosis as identified above. However, there is no detailed information available yet on the clinical trials using the HDAC inhibitors for the treatment of atherosclerosis in humans. Additional studies emphasizing the specific role of each HDAC and their effects on different cell types during the progression of atherosclerosis are needed. The ultimate outcome of such studies should substantiate the need for extensive evaluation of HDAC inhibitors through clinical trials which are currently lacking.

Technological advancements and possible applications. Improvements in sequencing strategies, mass spectrometry, Chromatin immunoprecipitation, microarray and other novel techniques have contributed to substantial progress in health science research. Increasing applications of these technological advancements would have positive effects in the development of treatment strategies that target de novo epigenetic changes in different disease conditions including atherosclerosis. Time-resolved NMR spectroscopy was innovatively developed to characterize asymmetric histone PTMs (71). Chromatin immunoprecipitation in conjunction with sequencing methodologies are typically used for mapping histone modifications. Through inclusion of micrococcal nuclease (MNase) digestion and barcoding, disease specific signatures can be identified at the single-cell level. Moreover, single-cell DamID can also be performed for genome-wide analysis of histone modifications using a cell line expressing Escherichia coli deoxyadenosine methylase (Dam) and combining it with specific histone readers or modifiers (72).

\section{Acknowledgements}

Not applicable.

\section{Funding}

The present review was developed with the funding support from 'LB692-Nebraska Tobacco Settlement Biomedical Research Development New Initiative Grants'.

\section{Availability of data and materials}

Not applicable.

\section{Authors' contributions}

WJ wrote the manuscript and performed the literature review. CSB developed the format of the review and corrected the manuscript. DKA assisted with the scientific content of the review and edited the manuscript.

\section{Ethics approval and consent to participate}

Not applicable.

\section{Consent for publication}

Not applicable.

\section{Competing interests}

The authors declare that they have no competing interests.

\section{References}

1. Seneviratne A, Hulsmans M, Holvoet $\mathrm{P}$ and Monaco C: Biomechanical factors and macrophages in plaque stability. Cardiovasc Res 99: 284-293, 2013.

2. Karlić R, Chung HR, Lasserre J, Vlahovicek K and Vingron M: Histone modification levels are predictive for gene expression. Proc Natl Acad Sci USA 107: 2926-2931, 2010.

3. Dong $\mathrm{X}$ and Weng Z: The correlation between histone modifications and gene expression. Epigenomics 5: 113-116, 2013.

4. Greer EL and Shi Y: Histone methylation: A dynamic mark in health, disease and inheritance. Nat Rev Genet 13: 343-357, 2012.

5. Xiaoling Y, Li Z, ShuQiang L, Shengchao M, Anning Y, Ning D, Nan L, Yuexia J, Xiaoming Y, Guizhong L and Yideng J: Hyperhomocysteinemia in ApoE-/-Mice leads to overexpression of enhancer of zeste homolog 2 via miR-92a regulation. PLoS One 11: e0167744, 2016.

6. Li Z, Cao R, Wang M, Myers MP, Zhang Y and Xu R: Structure of a Bmi-1-ring1B polycomb group ubiquitin ligase complex. J Biol Chem 281: 20643-20649, 2006.

7. Wierda RJ, Rietveld IM, van Eggermond MC, Belien JA, van Zwet EW, Lindeman JH and van den Elsen PJ: Global histone H3 lysine 27 triple methylation levels are reduced in vessels with advanced atherosclerotic plaques. Life Sci 129: 3-9, 2015.

8. Kadakol A, Malek V, Goru SK, Pandey A and Gaikwad AB: Esculetin reverses histone $\mathrm{H} 2 \mathrm{~A} / \mathrm{H} 2 \mathrm{~B}$ ubiquitination, $\mathrm{H} 3$ dimethylation, acetylation and phosphorylation in preventing type 2 diabetic cardiomyopathy. J Funct Foods 17: 127-136, 2015.

9. Xiao Y, Huang W, Zhang J, Peng C, Xia M and Ling W: Increased plasma $\mathrm{S}$-adenosylhomocysteine-accelerated atherosclerosis is associated with epigenetic regulation of endoplasmic reticulum stress in apoE-/-mice. Arterioscler Thromb Vasc Biol 35: 60-70, 2015.

10. Yuan Q, Xie X, Fu Z, Ma X, Yang Y, Huang D, Liu F, Dai C and Ma Y: Association of the histone-lysine N-methyltransferase MLL5 gene with coronary artery disease in Chinese Han people. Meta Gene 2: 514-524, 2014.

11. Chen WL, Sun HP, Li DD, Wang ZH, You QD and Guo XK: G9a-an appealing antineoplastic target. Curr Cancer Drug Targets 17: 555-568, 2017.

12. Cong G, Yan R, Huang H, Wang K, Yan N, Jin P, Zhang N, Hou J, Chen D and Jia S: Involvement of histone methylation in macrophage apoptosis and unstable plaque formation in methionine-induced hyperhomocysteinemic ApoE-/-mice. Life Sci 173: 135-144, 2017.

13. Zheng QF, Wang HM, Wang ZF, Liu JY, Zhang Q, Zhang L, Lu YH, You H and Jin GH: Reprogramming of histone methylation controls the differentiation of monocytes into macrophages. FEBS J 284: 1309-1323, 2017.

14. Greißel A, Culmes M, Burgkart R,Zimmermann A, Eckstein HH, Zernecke A and Pelisek J: Histone acetylation and methylation significantly change with severity of atherosclerosis in human carotid plaques. Cardiovasc Pathol 25: 79-86, 2016.

15. Bekkering $S$, van den Munckhof I, Nielen T, Lamfers E, Dinarello C, Rutten J, de Graaf J, Joosten LA, Netea MG, Gomes ME and Riksen NP: Innate immune cell activation and epigenetic remodeling in symptomatic and asymptomatic atherosclerosis in humans in vivo. Atherosclerosis 254: 228-236, 2016.

16. Vaquero A, Scher M, Erdjument-Bromage H, Tempst P, Serrano L and Reinberg D: SIRT1 regulates the histone methyl-transferase SUV39H1 during heterochromatin formation. Nature 450: 440, 2007. 
17. Li MF, Zhang R, Li TT, Chen MY, Li LX, Lu JX and Jia WP High glucose increases the expression of inflammatory cytokine genes in macrophages through $\mathrm{H} 3 \mathrm{~K} 9$ methyltransferase mechanism. J Interferon Cytokine Res 36: 48-61, 2016.

18. Paneni F, Costantino S, Battista R, Castello L, Capretti G Chiandotto S, Scavone G, Villano A, Pitocco D, Lanza G, et al: Adverse epigenetic signatures by histone methyltransferase Set7 contribute to vascular dysfunction in patients with type 2 diabetes. Circ Cardiovasc Genet 8: 150-158, 2015

19. Bekkering S, Quintin J, Joosten LA, van der Meer JW, Netea MG and Riksen NP: Oxidized low-density lipoprotein induces long-term proinflammatory cytokine production and foam cell formation via epigenetic reprogramming of monocytessignificance. Arterioscler Thromb Vasc Biol 34: 1731-1738, 2014.

20. Choi J, Yoon S, Kim S and Ahn Jo S: KDM4B histone demethylase and G9a regulate expression of vascular adhesion proteins in cerebral microvessels. Sci Rep 7: 45005, 2017.

21. Barroso M, Kao D, Blom HJ, Tavares De Almeida I, Castro R, Loscalzo J and Handy DE: S-adenosylhomocysteine induces inflammation through $\mathrm{NF \kappa B}$ : A possible role for $\mathrm{EZH} 2$ in endothelial cell activation. Biochem Biophys Acta 1862: 82-92, 2016.

22. Choi J and Jo SA: KDM7A histone demethylase mediates TNF- $\alpha$-induced ICAM1 protein upregulation by modulating lysosomal activity. Biochem Biophys Res Commun 478 : 1355-1362, 2016

23. Balcerczyk A, Rybaczek D, Wojtala M, Pirola L, Okabe J and El-Osta A: Pharmacological inhibition of arginine and lysine methyltransferases induces nuclear abnormalities and suppresses angiogenesis in human endothelial cells. Biochem Pharmacol 121: 18-32, 2016.

24. Gu L, Hitzel J, Moll F, Kruse C, Malik RA, Preussner J, Looso M, Leisegang MS, Steinhilber D, Brandes RP and Fork C: The histone demethylase PHF8 is essential for endothelial cell migration. PLoS One 11: e146645, 2016.

25. Han P, Gao D, Zhang W, Liu S, Yang S and Li X: Puerarin suppresses high glucose-induced MCP-1 expression via modulating histone methylation in cultured endothelial cells. Life Sci 130: 103-107, 2015.

26. Zernecke A: Dendritic cells in atherosclerosis. Arterioscler Thromb Vasc Biol 35: 763-770, 2015.

27. Kumar A, Kumar S, Vikram A, Hoffman TA, Naqvi A, Lewarchik CM, Kim YR and Irani K: Histone and DNA methylation-mediated epigenetic downregulation of endothelial kruppel-like factor 2 by low-density lipoprotein cholesterol. Arterioscler Thromb Vasc Biol 33: 1936-1942, 2013

28. Brasacchio D, Okabe J, Tikellis C, Balcerczyk A, George P, Baker EK, Calkin AC, Brownlee M, Cooper ME and El-Osta A: Hyperglycemia induces a dynamic cooperativity of histone methylase and demethylase enzymes associated with gene-activating epigenetic marks that coexist on the lysine tail. Diabetes 58 1229-1236, 2009.

29. El-Osta A, Brasacchio D, Yao D, Pocai A, Jones PL, Roeder RG, Cooper ME and Brownlee M: Transient high glucose causes persistent epigenetic changes and altered gene expression during subsequent normoglycemia. J Exp Med 205: 2409-2417, 2008.

30. Chen J, Zhang J, Yang J, Xu L, Hu Q, Xu C, Yang S and Jiang H: Histone demethylase KDM3a, a novel regulator of vascular smooth muscle cells, controls vascular neointimal hyperplasia in diabetic rats. Atherosclerosis 257: 152-163, 2017.

31. Yousefipour Z, Newaz MA, Esmaeilli M and Ranganna K: [PP.36.07] Modification of histone induced by acrolein in rat vascular smooth muscle cells. J Hypertens 34: e337, 2016.

32. Lehrke M, Kahles F, Makowska A, Tilstam PV, Diebold S Marx J, Stöhr R, Hess K, Endorf EB, Bruemmer D, et al: PDE4 inhibition reduces neointima formation and inhibits VCAM-1 expression and histone methylation in an Epac-dependent manner. J Mol Cell Cardiol 81: 23-33, 2015.

33. Qi H, Jing Z, Xiaolin W, Changwu X, Xiaorong H, Jian Y, Jing C and Hong J: Histone demethylase JMJD2A inhibition attenuates neointimal hyperplasia in the carotid arteries of balloon-injured diabetic rats via transcriptional silencing: Inflammatory gene expression in vascular smooth muscle cells. Cell Physiol Biochem 37: 719-734, 2015.

34. Villeneuve LM, Reddy MA, Lanting LL, Wang M, Meng L and Natarajan R: Epigenetic histone H3 lysine 9 methylation in metabolic memory and inflammatory phenotype of vascular smooth muscle cells in diabetes. Proc Natl Acad Sci USA 105: 9047-9052, 2008.
35. Xia J, Fang M, Wu X, Yang Y, Yu L, Xu H, Kong H, Tan Q, Wang $\mathrm{H}$, Xie $\mathrm{W}$ and $\mathrm{Xu} \mathrm{Y}$ : A2b adenosine signaling represses CIITA transcription via an epigenetic mechanism in vascular smooth muscle cells. Biochim Biophys Acta 1849: 665-676, 2015.

36. Tarling EJ, Ryan KJ, Austin R, Kugler SJ, Salter AM and Langley-Evans SC: Maternal high-fat feeding in pregnancy programs atherosclerotic lesion size in the ApoE*3 Leiden mouse. J Dev Orig Health Dis : Feb 2, 2016 (Epub ahead of print).

37. Alkemade FE, van Vliet P, Henneman P, van Dijk KW, Hierck BP van Munsteren JC, Scheerman JA, Goeman JJ, Havekes LM, Gittenberger-de Groot AC, et al: Prenatal exposure to apoe deficiency and postnatal hypercholesterolemia are associated with altered cell-specific lysine methyltransferase and histone methylation patterns in the vasculature. Am J Pathol 176: 542-548, 2010.

38. Bannister AJ and Kouzarides T: Regulation of chromatin by histone modifications. Cell Res 21: 381-395, 2011.

39. Witt O, Deubzer HE, Milde T and Oehme I: HDAC family: What are the cancer relevant targets? Cancer Lett 277: 8-21, 2009.

40. Tikoo K, Patel G, Kumar S, Karpe PA, Sanghavi M, Malek V and Srinivasan K: Tissue specific up regulation of ACE2 in rabbit model of atherosclerosis by atorvastatin: Role of epigenetic histone modifications. Biochem Pharmacol 93: 343-351, 2015.

41. Chaturvedi P, Kalani A, Givvimani S, Kamat PK, Familtseva A and Tyagi SC: Differential regulation of DNA methylation versus histone acetylation in cardiomyocytes during $\mathrm{HHcy}$ in vitro and in vivo: An epigenetic mechanism. Physiol Genomics 46: 245-255, 2014.

42. Kumar A and Chauhan S: How much successful are the medicinal chemists in modulation of SIRT1: A critical review. Eur J Med Chem 119: 45-69, 2016.

43. Sathishkumar C, Prabu P, Balakumar M, Lenin R, Prabhu D, Anjana RM, Mohan V and Balasubramanyam M: Augmentation of histone deacetylase 3 (HDAC3) epigenetic signature at the interface of proinflammation and insulin resistance in patients with type 2 diabetes. Clin Epigenetics 8: 125, 2016.

44. Hoeksema MA, Gijbels MJ, Van den Bossche J, van der Velden S, Sijm A, Neele AE, Seijkens T, Stöger JL, Meiler S, Boshuizen MC, et al: Targeting macrophage Histone deacetylase 3 stabilizes atherosclerotic lesions. EMBO Mol Med 6: 1124-1132, 2014

45. Van den Bossche J, Neele AE, Hoeksema MA, de Heij F, Boshuizen MC, van der Velden S, de Boer VC, Reedquist KA and de Winther MPJ: Inhibiting epigenetic enzymes to improve atherogenic macrophage functions. Biochem Biophys Res Commun 455: 396-402, 2014

46. Xie X, Song X, Yuan S, Cai H, Chen Y, Chang X, Liang B and Huang D: Histone acetylation regulates orphan nuclear receptor NR4A1 expression in hypercholesterolaemia. Clin Sci (Lond) 129: 1151-1161, 2015.

47. Oksala NKJ, Seppälä I, Rahikainen R, Mäkelä KM, Raitoharju E, Illig T, Klopp N, Kholova I, Laaksonen R, Karhunen PJ, et al: Synergistic expression of histone deacetylase 9 and matrix metalloproteinase 12 in M4 macrophages in advanced carotid plaques. Eur J Vasc Endovasc 53: 632-640, 2017.

48. Smith JD: New role for histone deacetylase 9 in atherosclerosis and inflammation. Arterioscler Thromb Vasc Biol 34: 1798-1799, 2014.

49. Cao Q, Rong S, Repa JJ, Clair RS, Parks JS and Mishra N: Histone deacetylase 9 represses cholesterol efflux and generation of alternatively activated macrophages in atherosclerosis development. Arterioscler Thrombosis Vasc Biol 34: 1871-1879, 2014.

50. Salah Ud-Din IA, Tikhomirova A and Roujeinikova A: Structure and Functional diversity of GCN5-related n-acetyltransferases (GNAT). Int J Mol Sci 17: E1018, 2016.

51. Chistiakov DA, Orekhov AN and Bobryshev YV: Treatment of cardiovascular pathology with epigenetically active agents: Focus on natural and synthetic inhibitors of DNA methylation and histone deacetylation. Int J Cardiol 227: 66-82, 2017.

52. Vecellio M, Spallotta F, Nanni S, Colussi C, Cencioni C, Derlet A, Bassetti B, Tilenni M, Carena MC, Farsetti A, et al: The histone acetylase activator pentadecylidenemalonate $1 \mathrm{~b}$ rescues proliferation and differentiation in the human cardiac mesenchymal cells of type 2 diabetic patients. Diabetes 63: 2132-2147, 2014

53. Zeidler R, de Freitas Soares BL, Bader A and Giri S: Molecular epigenetic targets for liver diseases: Current challenges and future prospects. Drug Discov Today 22: 1620-1636, 2017. 
54. Miranda TB, Cortez CC, Yoo CB, Liang G, Abe M, Kelly TK, Marquez VE and Jones PA: DZNep is a global histone methylation inhibitor that reactivates developmental genes not silenced by DNA methylation. Mol Cancer Ther 8: 1579-1588, 2009.

55. Wierda RJ, Goedhart M, van Eggermond MC, Muggen AF, Miggelbrink XM, Geutskens SB, van Zwet E, Haasnoot GW and van den Elsen PJ: A role for KMT1c in monocyte to dendritic cell differentiation. Hum Immunol 76: 431-437, 2015.

56. Zhao Q, Li S, Li N, Yang X, Ma S, Yang A, Zhang H, Yang S, Mao C, Xu L, et al: miR-34a targets HDAC1-regulated H3K9 acetylation on lipid accumulation induced by homocysteine in foam cells. J Cell Biochem 118: 4617-4627, 2017.

57. Makino J, Ogasawara R, Kamiya T, Hara H, Mitsugi Y, Yamaguchi E, Itoh A and Adachi T: Royal jelly constituents increase the expression of extracellular superoxide dismutase through histone acetylation in monocytic thp-1 cells. J Nat Prod 79: 1137-1143, 2016.

58. Svennerholm K, Haney M, Biber B, Ulfhammer E, Saluveer O, Larsson P, Omerovic E, Jern S and Bergh N: Histone deacetylase inhibition enhances tissue plasminogen activator release capacity in atherosclerotic man. PLoS One 10: e121196, 2015.

59. Anderson SJ, Feye KM, Schmidt-McCormack GR, Malovic E, Mlynarczyk GSA, Izbicki P, Arnold LF, Jefferson MA, de la Rosa BM, Wehrman RF, et al: Off-target drug effects resulting in altered gene expression events with epigenetic and Quasi-Epigenetic origins. Pharmacol Res 107: 229-233, 2016.

60. Cedar $\mathrm{H}$ and Bergman Y: Linking DNA methylation and histone modification: Patterns and paradigms. Nat Rev Gene 10: 295-304, 2009.

61. Yang F, Huo XS, Yuan SX, Zhang L, Zhou WP, Wang F and Sun SH: Repression of the long noncoding RNA-LET by histone deacetylase 3 contributes to hypoxia-mediated metastasis. Mol Cell 49: 1083-1096, 2013

62. Chen R, Kong P, Zhang F, Shu YN, Nie X, Dong LH, Lin YL, Xie XL, Zhao LL, Zhang XJ and Han M: EZH2-mediated $\alpha$-actin methylation needs lncRNA TUG1 and promotes the cortex cytoskeleton formation in VSMCs. Gene 616: 52-57, 2017.

63. Tee AE, Ling D, Nelson C, Atmadibrata B, Dinger ME, Xu N, Mizukami T, Liu PY, Liu B, Cheung B, et al: The histone demethylase JMJD1A induces cell migration and invasion by up-regulating the expression of the long noncoding RNA MALAT1. Oncotarget 5: 1793-1804, 2014.
64. Carrer A, Parris JLD, Trefely S, Henry RA, Montgomery DC, Torres A, Viola JM, Kuo Y, Blair IA, Meier JL, et al: Impact of a high-fat diet on tissue Acyl-CoA and histone acetylation levels. J Biol Chem 292: 3312-3322, 2017.

65. Short JD, Tavakoli S, Nguyen HN, Carrera A, Farnen C, Cox LA and Asmis R: Dyslipidemic diet-induced monocyte 'priming' and dysfunction in non-human primates is triggered by elevated plasma cholesterol and accompanied by altered histone acetylation. Front Immunol 8: 958, 2017.

66. Friso S, Carvajal CA, Fardella CE and Olivieri O: Epigenetics and arterial hypertension: The challenge of emerging evidence. Transl Res 165: 154-165, 2015.

67. Høgh Kølbæk Kjær AS, Brinkmann CR, Dinarello CA, Olesen R, Østergaard L, Søgaard OS, Tolstrup M and Rasmussen TA: The histone deacetylase inhibitor panobinostat lowers biomarkers of cardiovascular risk and inflammation in HIV patients. AIDS 29: 1195-1200, 2015.

68. Choi JH, Nam KH, Kim J, Baek MW, Park JE, Park HY, Kwon HJ, Kwon OS, Kim DY and Oh GT: Trichostatin A exacerbates atherosclerosis in low density lipoprotein receptor-deficient mice. Arterioscler Thromb Vasc Biol 25: 2404-2409, 2005.

69. Okamoto H, Fujioka Y, Takahashi A, Takahashi T, Taniguchi T, Ishikawa $\mathrm{Y}$ and Yokoyama M: Trichostatin A, an inhibitor of histone deacetylase, inhibits smooth muscle cell proliferation via induction of p21 (WAF1). J Atheroscler Thromb 13: 183-191, 2006.

70. Pandey D, Sikka G, Bergman Y, Kim JH, Ryoo S, Romer L and Berkowitz D: Transcriptional regulation of endothelial arginase 2 by histone deacetylase 2. Arterioscler Thromb Vasc Biol 34: 1556-1566, 2014.

71. Liokatis S, Klingberg R, Tan S and Schwarzer D: Differentially isotope-labeled nucleosomes to study asymmetric histone modification crosstalk by time-resolved NMR spectroscopy. Angew Chem Int Ed Engl 55: 8262-8265, 2016.

72. Clark SJ, Lee HJ, Smallwood SA, Kelsey G and Reik W: Single-cell epigenomics: Powerful new methods for understanding gene regulation and cell identity. Genome Biol 17: 72, 2016. International (CC BY-NC-ND 4.0) License. 